\title{
Associação entre dores nas costas, flexibilidade e força/resistência abdominal em crianças e adolescentes
}

\author{
Association between back pain, flexibility and abdominal strength \\ in children and adolescents
}

Aline Carrer Bortolini,' Diogo Bezzi Jaeger,, Osvaldo Donizete Siqueira,' Luiz Antonio Barcellos Crescente, ${ }^{1}$ Daniel Carlos Garlipp ${ }^{1}$

'Universidade Luterana do Brasil (ULBRA/RS), Porto Alegre, RS, Brasil.

Recebido em: 05/07/2016 / Aceito em: 22/08/2016 / Publicado em: 30/09/2016

dcgarlipp@gmail.com

\section{RESUMO}

Objetivo: verificar se existe associação entre dores nas costas, baixa força/resistência abdominal e pouca flexibilidade. Método: foram avaliados um total de 162 crianças e adolescentes (79 meninos e 83 meninas), com idades entre os nove e os 15 anos de idade, de duas escolas da cidade de Canoas/RS. A força/resistência abdominal foi medida através do teste do sit up's (número de abdominais em um minuto) e a flexibilidade foi medida através do teste de sentar-e-alcançar $(\mathrm{cm})$, seguindo as recomendações do Projeto Esporte Brasil (Proesp). Com o uso de pontos de corte específicos por sexo e idade, a flexibilidade e a força/resistência abdominal foram classificadas em "zona de risco à saúde" e "zona saudável". A presença de dores nas costas foi medida através do relato dos alunos. Para a estatística descritiva, foi utilizada a média e o desvio padrão. Para a estatística inferencial, foi utilizado o teste do Qui-quadrado. Todas as análises foram realizadas no programa estatístico SPSS 20.0, com nível de significância de $5 \%$. Resultados: não foram identificadas, de forma isolada, associações estatisticamente significativas entre baixa força/resistência abdominal e dores nas costas, assim como baixa flexibilidade e dores nas costas. Também, ao serem analisados aqueles indivíduos que apresentavam conjuntamente baixa resistência/abdominal e baixa flexibilidade e relacionando a dores nas costas, também não foram identificadas associações estatisticamente significativas. Considerações finais: o relato de dores nas costas não parece estar associado a uma baixa força/resistência abdominal e a uma baixa flexibilidade.

Palavras-chave: Dor nas costas; Criança; Adoles-

\section{ABSTRACT}

Objective: checking if exist association between back pain and low abdominal strength and little flexibility. Method: was used a total of 162 students 179 male and 83 female), with ages between nine and 15 years old, from two different schools in Canoas/RS. The abdominal strength was measured by the sit-ups test (number of abdominals in a minute) and the flexibility was measured by sit and reach test $(\mathrm{cm})$, following the Projeto Esporte Brasil (Proesp) recommendations. Using specific cutoff points by sex and age, the flexibility and abdominal strength were classified as "risk zone to health" and "healthy zone". The back pain was measured by reporting of students. For descriptive statistic, it was used average and standard deviation. For inferential statistic, it was used Qui-quadrado test. All analysis were performed in the statistical program SPSS 20.0, being the level of significance adopted 5\%. Results: not identified, singly, statistically significant associations between low abdominal strength and back pain (male: $p=0,568$; female: $p=0,070$ ), even as little flexibility and back pain (male: $p=0,567$; female: $p=0,195$ ). Also, when analyzing those individuals who had jointly low abdominal strength and little flexibility and relating to back pain, were not statistically significant associations identified. Closing remarks: that back pain report does not seem to be associated to low abdominal strength and little flexibility.

Keywords: Back pain; Child; Adolescent. cente. 


\section{INTRODUÇÃO}

Um dos problemas que a população brasileira mais demonstra em relação à saúde é a lombalgia, ou seja, a dor nas costas. A lombalgia não é, necessariamente, uma doença e sim um sintoma que pode estar relacionado a uma doença, ${ }^{1,2}$ principalmente por envolver uma série de músculos, associados, ora com a estabilização, ora com a flexão, inclinação e rotação do corpo. Uma pessoa com dor nas costas se torna restrita ou até incapaz de realizar certos movimentos, podendo se prejudicar no trabalho, na escola e em suas atividades diárias. Se a estimativa da Organização Mundial da Saúde ${ }^{3}$ se confirmar, cerca de 80\% das pessoas terão, no mínimo, uma crise de dor lombar durante a vida e desses, $90 \%$ apresentarão repetição da dor.

Quando se pensa em dor nas costas em crianças, se questiona o fato de muitas delas terem sintomas de dor em uma idade tão precoce. Conforme Méndez e Gómez-Coneza, ${ }^{4}$ a prevalência de dores lombares em crianças de 9 a 10 anos é similar à da população adulta. Já, avaliando estudantes dinamarqueses, Wedderkopp et al. ${ }^{5}$ identificaram incidência crescente de dor, chegando a $47 \%$ nos adolescentes. As crianças podem ter ou desenvolver os fatores de risco para lombalgia, visto que são multifatoriais. Dentre os principais fatores para o desenvolvimento dessas dores está o sexo, peso, altura, mobilidade dos músculos, baixa flexibilidade, obesidade, peso e modo de carregar a mochila, postura incorreta e sedentarismo. ${ }^{6}$ Além disso, aptidão física e força e resistência abdominal também parecem ter relação com as dores nas costas. . $^{3,-9}$

Sabe-se que os músculos exercem diversas funções. A musculatura do abdome tem, além de suas funções de flexor do tronco (pelo reto abdominal e oblíquos internos e externos) e de rotação e inclinação lateral (pelos oblíquos internos e externos), uma participação importante na estabilização da coluna lombar e da cintura pélvica, principalmente durante o exercício abdominal. ${ }^{10-11}$ Desta forma, estudos confirmam a relação entre a baixa elasticidade dos músculos com dores lombares. ${ }^{3,8-9}$ Steene-Johannessen et al. ${ }^{12}$ sugerem haver uma relação inversa entre os níveis de aptidão física e fatores de risco para o desenvolvimento de lombalgia. Todavia, não há um consenso na literatura sobre a relação entre pouca flexibilidade e baixa força/resistência abdominal com dores nas costas.

Sendo assim, o objetivo do presente estudo foi verificar se existe associação entre dores nas costas com baixa força/resistência abdominal e pouca flexibilidade.

\section{MÉTODO}

Para o presente estudo, descritivo e associativo, com análise de corte transversal, foram avaliados um total de 135 alunos (69 do sexo masculino e 66 do sexo feminino), com idades entre os nove e os 15 anos de idade, de duas escolas da cidade de Canoas/RS.

A força/resistência abdominal foi medida através do teste do sit up's (número de abdominais em um minuto) enquanto que a flexibilidade foi medida através do teste de sentar-e-alcançar (sit-and-reach), seguindo as recomendações do Projeto Esporte Brasil. ${ }^{13} \mathrm{Com}$ o uso de pontos de corte específicos por sexo e idade, a flexibilidade e a força/resistência abdominal foram classificadas em "zona de risco à saúde" e "zona saudável".

Para a aprendizagem dos testes, todos os participantes receberam informações acerca dos procedimentos, observaram as demonstrações de ambos os testes, e então, foram submetidos a tentativas de cada teste no mesmo dia. Todas as medidas e testes foram realizados pelo mesmo pesquisador, no mesmo local, utilizando os mesmos equipamentos. Nas análises foi considerado o maior valor obtido nas tentativas de cada teste.

A presença de dores nas costas foi anotada conforme relato dos avaliados.

Para a estatística descritiva, foram utilizados os valores absolutos e em percentual. Para a estatística inferencial, foram utilizados a Correlação de Pearson e o teste do Qui-quadrado. Considerou-se um nível de significância de $5 \%(p<0,05)$. Todas as análises foram realizadas no programa estatístico SPSS, versão 20.0.

Só participaram do estudo os alunos que entregaram o Termo de Consentimento Livre e Esclarecido (TCLE), sendo que a pesquisa tem aprovação do Comitê de Ética da Universidade Federal do Rio Grande do Sul sob número 17916.

\section{RESULTADOS}

Os resultados são apresentados na tabela 1 onde, através dos valores críticos de flexibilidade e força/ resistência abdominal, ${ }^{13}$ foram classificados em zona saudável e zona de risco à saúde.

Quanto à flexibilidade, das 66 escolares do sexo feminino avaliadas, $31,8 \%$ foram classificadas como estando na "zona saudável". Já, no sexo masculino, somente $37,7 \%$ foram classificados como estando na "zona saudável". Em relação à força/resistência abdominal, a porcentagem de estudantes que ficou classificada como estando na "zona saudável" foi de 50,0\% nas meninas e $76,8 \%$ nos meninos

Em relação às dores nas costas, foi demonstrada uma prevalência de dor nas meninas de $13,6 \%$, enquanto que nos meninos esse percentual chegou a $8,7 \%$.

Não foi identificada correlação entre os resultados provenientes dos testes do sentar-e-alcançar e o teste do sit up's, tanto no sexo masculino $(p=0,433)$ como no sexo feminino $(p=0,892)$.

Tabela 1 - Classificação dos valores de flexibilidade e força/resistência abdominal nos dois sexos.

\begin{tabular}{|c|c|c|c|c|c|}
\hline \multirow[b]{2}{*}{ Variável } & \multirow[b]{2}{*}{ Sexo } & \multicolumn{2}{|c|}{ Zona Saudável } & \multicolumn{2}{|c|}{ Zona de Risco à Saúde } \\
\hline & & v.a. & v.p. & v.a. & v.p. \\
\hline \multirow[t]{2}{*}{ Flexibilidade } & Masculino & 26 & $37,7 \%$ & 43 & $62,3 \%$ \\
\hline & Feminino & 21 & $31,8 \%$ & 45 & $68,2 \%$ \\
\hline \multirow[t]{2}{*}{ Força/resistência abdominal } & Masculino & 53 & $76,8 \%$ & 16 & $23,2 \%$ \\
\hline & Feminino & 33 & $50,0 \%$ & 33 & $50,0 \%$ \\
\hline
\end{tabular}

v.a. $=$ valores absolutos; $v . p .=$ valores em percentual 
Não foram identificadas associações estatisticamente significativas entre as categorias de flexibilidade e os sexos $(p=0,475)$. Entretanto, foi identificada associação estatisticamente significativa entre o sexo feminino e estar na zona de risco à saúde e ser do sexo masculino e estar na zona saudável quanto à força/ resistência abdominal ( $p=0,001$; ajuste residual $=3,2)$.

Não foram identificadas associações estatisticamente significativas entre dor nas costas e os critérios da força/resistência abdominal tanto no sexo masculino $(p=0,159)$ quanto no sexo feminino $(p=0,720)$. Como não foram identificadas associações estatisticamente significativas entre dor nas costas e os critérios da flexibilidade tanto no sexo masculino $(p=0,266)$ quanto no sexo feminino $(p=0,506)$.

\section{DISCUSSÃO}

Conforme Achour Júnior, ${ }^{14}$ o teste de sentar-e-alcançar vêm sendo um dos mais indicados, para a avaliação da flexibilidade, pelo fato de avaliar a flexibilidade na coluna e nos músculos isquiotibiais, que estão intimamente associados à grande parte das reclamações de dores nas costas e aos problemas de postura. ${ }^{14}$

Em um estudo desenvolvido por Pereira et al., ${ }^{15}$ na região Centro-Oeste do país, aproximadamente $80 \%$ dos analisados que obtiveram bons resultados para flexibilidade eram meninas. Embora alguns autores não tenham observado diferenças estatisticamente significativas na flexibilidade entre os sexos, nas diferentes idades, ${ }^{16-18}$ as meninas foram mais flexíveis que os meninos em muitos achados, ${ }^{19-20,22-23}$ corroborando com o encontrado no presente estudo. Para Bernard et al., ${ }^{24}$ dos 6 aos 12 anos de idade, os meninos tendem a apresentar um desempenho mais satisfatório nas tarefas motoras que exigem potência muscular, enquanto que as meninas se destacam naquelas voltadas ao equilíbrio e flexibilidade. Segundo Heyward, ${ }^{25}$ as meninas serão mais flexíveis que os meninos, não importa a idade, devido aos hormônios e à estrutura anatômica.

Em relação à força/resistência abdominal, em outros estudos, o sexo masculino apresenta um desempenho melhor nos testes. ${ }^{9,14,20}$

Em um estudo com crianças e adolescentes feitas na população geral, avaliando a prevalência de dor lombar, Balagué et al. ${ }^{2}$ demonstraram resultados entre $30 \%$ e $51 \%$. Quanto ao sexo, em outros estudos, as meninas apresentaram maiores queixas de dores nas costas do que os meninos. ${ }^{26-31}$ Possivelmente, as diferenças entre os sexos podem ocorrer devido a uma maior força física apresentada pelos meninos, o estado psicológico e social onde as meninas seriam mais frágeis emocionalmente, ${ }^{26,32}$ a postura e a fraqueza dos músculos abdominais. . $^{8,33}$

Em estudos realizados com adolescentes finlandeses, cada vez mais há relatos de dores no pescoço, no ombro e na região lombar, sugerindo uma possível doença osteomuscular degenerativa em adultos no futuro. Essa dor, como nos outros estudos citados, foi mais comum entre as meninas..$^{1,30}$

Quanto a não existência de correlação estatisticamente significativa entre flexibilidade e força-resistência abdominal, comparativamente a outros estudos, são identificadas divergências, visto que a musculatura dorsal tem apresentado interferências nas musculaturas abdominal e das coxas. Desta forma, os testes apresentam valores mais elevados quanto maior for a capacidade de extensão dos quadris, da coluna e da parte posterior das coxas. ${ }^{33}$

Quanto à relação de flexibilidade com dores nas costas, estudos apresentaram associação, demonstrando que indivíduos com boa flexibilidade tem menor chance de ter lesões musculoesqueléticas ${ }^{21}$ e dores musculares (especialmente lombares), além de ser uma prevenção contra complicações na postura. ${ }^{14} \mathrm{Em}$ um estudo realizado por 25 anos, a flexibilidade, em um nível adequado, durante o período da adolescência resultou em menores níveis de dores cervicais na idade adulta. ${ }^{34}$ Em um estudo desenvolvido por Comerford e Mottram $^{35}$, foi identificado que todas as crianças que relataram dores lombares apresentavam baixa flexibilidade. Por outro lado, outros estudos não identificaram qualquer associação entre flexibilidade e dores nas costas. . $^{8,35}$

Em relação a não existência de associações estatisticamente significativas entre dor nas costas e força e resistência abdominal, um estudo com adultos, realizado na Austrália, todos os pacientes com dores nas costas analisados possuíam a musculatura profunda do abdome fraca e uma sobrecarga de músculos globais. ${ }^{35}$ Essa sobrecarga é tida como resultado de uma compensação, ou seja, quando os músculos profundos do abdome não exercem adequadamente suas funções, os músculos globais os substituem. ${ }^{3}$ Desta forma, os exercícios abdominais são sugeridos com uma forma de prevenção ao desenvolvimento de dores lombares. ${ }^{36}$

Enfim, os resultados desse estudo demonstram que relações que estão bem estabelecidas na idade adulta ainda precisam de mais investigações quando se trata de crianças e adolescentes.

\section{CONSIDERACÕES FINAIS}

Identificar as possíveis relações entre o relato de dores nas costas em crianças e adolescentes com outras variáveis é de fundamental importância no que tange ao entendimento dos diversos fatores de risco, a fim de que se previnam doenças que tragam mais acometimentos ao indivíduo adulto. Quando relacionada à baixa flexibilidade e à pouca força/resistência abdominal com o relato de dores nas costas, não se identificou associações estatisticamente significativas, tanto para o sexo masculino, quanto para o feminino.

Ressalta-se, no entanto, que por mais estudos que se tenha a respeito dessa condição, ainda se faz necessária a realização de trabalhos mais profundos, a fim de que haja uma maior compreensão do assunto.

\section{REFERÊNCIAS}

1. Hakala P, Rimpela A, Salminen JJ, Virtanen SM, Rimpela M. Back, neck, and shoulder pain in Finnish adolescents: national cross sectional surveys. BMJ 2002;325(7367):743.

2. Balagué $F$, Troussier B, Salminen JJ. Non-specific low back pain in children and adolescents: risk factors. Eur Spine $J$ 
1999;8(6):429-38.

3. Gouveia K, Gouveia E. O músculo transverso abdominal e sua função de estabilização da coluna lombar. Fisioter Mov 2008;21(3):45-50.

4. Méndez FJ, Gómez-Coneza A. Postural hygiene program to prevent low back pain. Spine 2001;26:1280-6.

5. Wedderkopp N, Leboeuf-Yde De C, Andersen LB, Karsten $F$, Hansen HS. Back pain reporting pattern in a Danish population-based sample of children and adolescents. Spine 2001;26:1879-83.

6. Cardon G, Balagué F. Low back pain prevention's effects in schoolchildren: what is the evidence? Eur Spine J 2004; 13:663-79. doi: 10.1007/s00586-004-0749-6.

7. Lemos A, Santos F, Moreira R, Machado D, Braga F, Gaya A. Ocorrência de dor lombar e fatores associados em crianças e adolescentes de uma escola privada do sul do Brasil. Cad Saúde Pública. Rio de Janeiro 2013;29(11):2177-2185.

8. Graup S, Bergmann ML, Bergmann GG. Prevalência de dor lombar inespecífica e fatores associados em adolescentes de Uruguaiana/RS. Rev Bras Ortop 2014;49(6):661-667. doi: 10.1016/j.rbo.2013.09.005.

9. Guedes $D$, Guedes JE. Aptidão física relacionada à saúde de crianças e adolescentes: avaliação referenciada por critério. Rev Bras Ativ Fís Saúde 1995;1(2):27-38.

10. Mirka GA. Selective recruitment of external oblique during axial torque production. Clin Biomech 1997;(67):1213-17.

11. Kapandji Al. Fisiologia articular: tronco e coluna vertebral. $5^{a}$ ed. Rio de Janeiro: Guanabara Koogan; 2000.

12. Steene-Johannessen, J; Anderssen, SA; Kolle, E; Andersen, LB. Low Muscle Fitness Is Associated with Metabolic Risk in Youth. Med Sci Sport Exer. Hagerstown 2009;41(17):1361 67. doi: 10.1249/MSS.0b013e31819aaae5

13. Projeto Esporte Brasil: manual. Disponível em: <https:// www.proesp.ufrgs.br> Acesso em: 10 setembro 2015.

14. 14. Achour Júnior $A$. Bases para o exercício de alongamento relacionado com a saúde e no desempenho atlético. Londrina, PR: Midiograf, 1996.

15. Pereira C, Ferreira D, Copetti G, Guimarães L, Barbacena M, Liggeri N, Castro O, Lobato S, David AC. Aptidão física em escolares de uma unidade de ensino da rede pública de Brasília-DF. Rev Bras Ativ Fís Saúde 2011;16(3):223-7.

16. Araujo $S$, De Oliveira AC. Aptidão física em escolares de Aracaju. Rev Bras Cineantropom Desempenho Hum 2008;10(3):271-6.

17. Ribeiro C, Abad C, Barros R, Barros Neto T. Nível de flexibilidade obtida pelo teste de sentar-e-alcançar a partir de estudo realizado na Grande São Paulo. Rev Bras Cineantropom Desempenho Hum 2010;12(6):415-21.

18. Da Silva D, Dos Santos JA, De Oliveira BM. A flexibilidade em adolescentes - um contributo para a avaliação global. Rev Bras Cineantropom Desempenho Hum 2006;8(1):72-9.

19. Andreasi $V$, Michelin $E$, Rinaldi $A E$, Burini R. Aptidão física associada às medidas antropométricas de escolares do ensino fundamental. J Pediatr. Rio de Janeiro 2010;86(6): 497-502. doi: $10.1590 /$ S0021-75572010000600009

20. Rassilan E, Guerra T. Evolução da flexibilidade em crianças de 7 a 14 anos de idade de uma escola particular do município de Timóteo-MG. Movimentum - Revista Digital de Educação
Física. Ipatinga, MG 2006;1:1-13.

21. Hoeger WK, Hoeger SA. Principles and Labs for Physical Fitness and Wellnem. Colorado. USA: $3^{\mathrm{a}}$ ed. Morton Pub Co: 1994.

22. Malina, R.M. \& Bouchard, C. Growth, maturation and physical activity. Champaign, Human Kinetics Books, 1991.

23. De Vitta $A$, Martinez $M$, Piza $N$, Simeão $S$, Ferreira $N$. Prevalência e fatores associados à dor lombar em escolares. Cad Saúde Pública. Rio de Janeiro 2011;27(8):1520-8. doi: 10.1590/S0102-311X2011000800007.

24. Bernard JC, Bard R, Pujol A, Combev A, Boussard D, Begue $C$, et al. Muscle assessment in healthy teenagers comparison with teenagers with low-back pain. Ann Readapt Med Phys 2008;51(4):274-83. doi: 10.1016/j.annrmp.2008.03.010

25. Heyward VH. Advanced Fitness Assessment Exercise Prescription. 3 ed. United States: Human Kinetics, 1998.

26. Bo Andersen L, Wedderkopp N, Leboeuf YC. Association between back pain and physical fitness in adolescents. Spine 2006;31(15):1740-4.

27. Swain M, Henschke N, Kamper S, Gobina I, Ottová-Jordan V, Maher C. An international survey of pain in adolescents. BMC Public Health 2014;14(447):1471-2458. doi: 10.1186/14712458-14-447.

28. Wedderkopp N, Andersen L, Froberg K, Leboeuf-YDE C. Back pain reporting in young girls appears to be puberty related. BMC Musculoskeletal Disorders 2005;6(52):1471-4. doi: 10.1186/1471-2474-6-52.

29. Wirth B, Knecht C, Humphreys K. Spinal pain in Swiss school children - epidemiology and risk factors. BMC Pediatrics 2013;13(159):1471-2431.

30. Maciel SC, Jennings F, Jones A, Natour J. The development and validation of a low back pain knowledge questionnaire. LKO Clinics 2009;64(12):1167-75. doi: 10.1590/S180759322009001200006.

31. Guedes D. Implicações associadas ao acompanhamento do desempenho motor de crianças e adolescentes. Rev Bras Educ Fís Esp. São Paulo 2007;21:37-60. doi: 10.1590/ S1807-55092007000500005.

32. Mikkelson LO, Nupponen $\mathrm{H}$, Kaprio J, Kautiainen $\mathrm{H}$, Mikkelson $\mathrm{M}$, Kujala UM. Adolescent flexibility, endurance strength, and physical activity as predictors of adult tension neck, low-back pain, and knee injury: a 25-year follow up study. $\mathrm{Br} \mathrm{J}$ Sports Med 2006;40:107-13. doi: 10.1136/bjsm.2004.017350.

33. Penha P, Amado João SM. Avaliação da flexibilidade muscular entre meninos e meninas de 7 e 8 anos. Fisioter e Pesq 2008; 15(4):387-91. doi: 10.1590/S1809-29502008000400012.

34. Cardon G, Boudeaudhuij LD, Clercq DD, Philippaerts R, Verstraeste S, Geldhof E. Physical fitness, physical activsity, and self-reported back and neck pain in elementary school children. Pediatr Exerc Sci 2004;16:147-57.

35. Comerford MJ, Mottram SL. Movement and stability dysfunction - contemporary developments. Man Ther 2001; 6(1):15-26. doi: 10.1054/math.2000.0388.

36. Rodacki CLN, Fowler NE, Rodacki ALF, Birch K. Stature loss and recovery in pregnant women with and without low back pain. Arch Phys Med Rehab 2003;84:507-512. doi: 10.1053/ apmr.2003.50119.

Como citar: BORTOLINI, Aline Carrer et al. Associação entre dores nas costas, flexibilidade e força/resistência abdominal em criancas e adolescentes. Cinergis, Santa Cruz do Sul, v. 17, n. 3, set. 2016. ISSN 2177-4005. Disponível em: <https://online.unisc.br/seer/index.php/cinergis/article/view/7797 >. Acesso em: 30 set. 2016. doi:http://dx.doi.org/10.17058/cinergis. v17i3.7797. 